\title{
A Mechanistic-Empirical Based Overlay Design Method for Reflective Cracking
}

\author{
Jorge B. Sousa*, Jorge C. Pais ${ }^{* *}$, George B. Way*** \\ * Consulpav International \\ 724 Laurel Drive, Walnut Creek, CA 94596, USA \\ jmbsousa@aol.com \\ ** University of Minho \\ 4800-058 Guimarães, Portugal \\ jpais@civil.uminho.pt \\ *** Arizona Department of Transportation \\ Materials Group, MD 068, 1221 N. 21st Ave., Phoenix, Arizona 85009, USA \\ gway@dot.state.az.us
}

\begin{abstract}
This paper describes a new and innovative mechanistically based pavement overlay design method that considers the most predominant type of overlay distress observed in the field: Reflective cracking above old cracks in the underlying pavement surface. Both dense-graded hot mix asphalt and gap-graded asphalt rubber (wet process) mixes were studied, in the laboratory and in the field, to derive the necessary mechanistic relationships and statistically based equations. The models proposed are based on a finite element model that closely approximates actual field phenomena. Many field test sections, in Arizona, California and Portugal, were studied during the course of the research.

Other HMA mixes used for overlays may also be calibrated and used through the proposed method. However, the relevant mix properties of any additional materials or environmental zones must first be determined. The two mix types studied are mainly used in the desert southwest region of Arizona and California. The overlay design program is available from the Rubber Pavements Association or Arizona Department of Transportation in the form of an Excel spreadsheet with an easy-to-use visual basic computer program (macro).
\end{abstract}

KEY WORDS: Reflective Cracking, Overlay Design, Fatigue Test 


\section{Introduction}

The Rubber Pavements Association (RPA) contracted with Consulpav in 1999 to develop a mechanistic overlay design method for reflective cracking. Reflective cracking is cracking that occurs in a hot mix asphalt (HMA) overlay after it has been applied to an existing cracked paved surface. The existing pavement may be either HMA or Portland Cement Concrete (PCC). RPA's interest in sponsoring this research effort was based upon the long-standing observation that asphalt rubber (AR) hot mixes appear to reduce the occurrence of reflective cracking. Also, there appeared to be a need to develop a mechanistic reflective cracking model and overlay design method to complement the new 2002 Pavement Design Guide, which will focus on mechanistic methods of pavement design.

This paper is a condensed version of an eight chapter, 200-page report, entitled "Development of a Mechanistic Overlay Design Method Based on Reflective Cracking Concepts" [SOU 2001]. That report and all supporting information is available, free of charge, on a CDROM from the Arizona Department of Transportation or from the RPA.

Consulpav performed its research in cooperation and coordination with the University of Minho in Portugal and the Arizona Department of Transportation (ADOT). ADOT's interest stems from its extensive use of AR overlays on hundreds of state projects in order to reduce reflective cracking [WAY 79], [SCO 89], [WAY 89] and [WAY 2000]. Both Portugal and Arizona perceived a need to develop a mechanistic approach to the design of AR-HMA and conventional HMA overlays, which could be of help to them in their future use of the mechanistically based 2002 AASHTO Pavement Design Guide.

The research project first involved the development of a model based on the Finite Element Method (FEM). The FEM approach was selected since it appeared to be the most sensible way to address the unusual stress and strain contours generated by a heavy wheel load moving over or near a crack. To calibrate the FEM-modeled crack movements, actual field measurements with a Crack Activity Meter (CAM) and a Falling Weight Deflectometer (FWD) were both conducted, in Portugal, Arizona and California. The majority of the field-testing was conducted on cracked highway pavements in Arizona. Results of this testing helped to calibrate the FEM model and showed that the CAM was well correlated to FWDinduced crack movements and deflections.

The next phase of the research involved laboratory testing to simulate observed field crack movements and measure stresses and strains in test specimens to simulate actual field conditions. Two typical mixes using a good quality Portuguese granite aggregate similar to Watsonville, California's granite aggregate were prepared and tested in Portugal. One conventional HMA mix consisted of a dense graded aggregate similar to that used in Arizona and California, hereinafter called HMADG. Five percent PG 70-10 asphalt was added to the dense graded aggregate mix and compacted using the rolling wheel compactor to various air void levels. The second mix consisted of an eight percent AR-HMA gap-graded mix (hereinafter 
called AR-HMA-GG) similar to those used in Arizona and California. The AR binder consisted of 80\% PG 64-16 (Penetration 35/50) base asphalt and 20\% ground tire rubber. These HMA-DG and AR-HMA-GG mixes represent the basis of the proposed mechanistic reflective cracking overlay design method.

Cores and beams were sawed from the rolling wheel compacted slabs. Each 150 $\mathrm{mm}$ (six-inch) diameter by $50 \mathrm{~mm}$ (two-inch) thick core was tested with a "reflective crack" testing device (RCD), specifically designed and built for use in this research project. This device is designed with a preset crack, which the circular core is placed directly above. The core is clamped in such a way that either (or both) a horizontal or vertical movement can be imposed on the core, above the crack. Sensors on the core record stresses and strains of the core immediately above the crack. In addition, beams of HMA-DG and AR-HMA-GG were tested with the fourpoint bending beam fatigue test developed during the Strategic Highway Research Program (SHRP). Results of these tests indicated that beam fatigue test measurements could be used in place of the RCD to derive the necessary input parameters to the FEM model. With this knowledge, it was now possible to determine which parameters best fit the FEM reflective cracking statistical simulation as a function of heavy truck traffic. The model predicts how many heavy wheel loads and their attendant stresses and strains are needed to initiate and propagate a reflective crack.

To convert this mathematical statistical model into a practical pavement design method for reflective cracking, it was necessary to review considerable actual field cracking data and material layer properties. From these data, the estimated traffic to cause reflective cracking was calculated from the layer thicknesses and layer moduli in a variety of pavements. These calculated numbers were compared to both the actual (observed) number of equivalent axle loads and the (observed) percent cracking. A very novel relationship was derived, which indicates that as long as the ratio between the estimated and actual traffic to cause reflective cracking stays below one, no cracking will occur. For ratios above one, different levels of percent cracking are calculated and observed. Aging and temperature adjustment factors were also a novel adjunct to this new approach.

The final product of this research is a spreadsheet where the pavement design engineer inputs the expected design level of cracking, the thicknesses of the layers, and their elastic modulus. The modulus may be backcalculated or determined in any reasonable manner, as long as they represent the in situ conditions in the field. The resultant curves, one for the PG 70-10 HMA-DG and one for the AR-HMA-GG mix, estimate the thickness of an overlay for the specified level of reflective cracking, over a wide range of truck traffic loadings. To-date, the proposed method mainly applies to these two mix types, for climatic conditions similar to those encountered in the (mainly) desert southwest.

With additional research, other overlay design curves can be developed for other mixes, other climates, and other field-observed historical reflective cracking levels. This research describes the development of a mechanistic reflective cracking model based upon a FEM approach that can also be used for other materials and climates. 


\section{Crack Modelling}

This section describes a FEM of modeling the movement (and, the stresses and strains) through an overlay in the pavement immediately above a crack. The FEM used the 3D linear elastic model based on the SAP2000 software.

An effort was made to make this simulation as close as practicably possible to field observations. From this simulation, a statistically generalized (albeit simplified) mathematical expression has been developed for the specific materials and layer thicknesses involved. The reason for offering a simplified statistical approach is that FEM analyses are very difficult and time consuming, thus they are probably outside the scope of routine use by the typical pavement design engineer.

A statistical approach appears reasonable for the limited data and special boundary conditions implied from actual field measurements. Thus, to some degree, the proposed mechanistic design method for reflective cracking does have an empirical component. This is not uncommon, since the stress/strain condition within a cracked pavement is very difficult to measure and model. Statistical methods can only provide exact answers in the simplest of cases. To extend their use to more realistic calculations, it is usually necessary to provide some empirical information to substantiate the theoretical model. As such, the use of statistical models that can be incorporated into easy-to-use spreadsheets such as EXCEL renders a complex, detailed and mechanistic pavement rehabilitation design approach available to all.

\subsection{Measured vs. Predicted Crack Activity before Overlay}

The first effort to verify and calibrate the purely FEM mechanical analysis of a cracked pavement before overlay developed was accomplished by comparing measured versus calculated vertical crack movements.

This FEM model was used to compute the vertical crack activity, before overlay, that was fitted in the statistical model presented in Equation [1].

$$
A c t=a \cdot \log (\text { Tbet })+b \cdot \log (\text { Tgra })+c \cdot \log (\text { Mbet })+d \cdot \log (\text { Mgra })+e \cdot \log (\text { Msub })+f
$$

Where:

Act $=$ vertical crack activity before overlay $\left(10^{-6} \mathrm{~m}\right)$

Tbet $=$ bituminous cracked layer thickness (m)

Tgra $=$ granular layer thickness $(\mathrm{m})$

Mbet $=$ bituminous cracked layer modulus $(\mathrm{MPa})$

Mgra = granular layer modulus $(\mathrm{MPa})$

Msub = subgrade modulus $(\mathrm{MPa})$

Constants: $a=11.773 ; b=-21.388 ; c=-52.893 ; d=-94.619 ; e=16.418$ and

$\mathrm{f}=1181.660\left(\mathrm{R}^{2}=0.92\right)$ 
Layer moduli from backcalculation and the layer thicknesses obtained from seven cracked test sections in Arizona and California were used in the statistical model derived from a purely FEM mechanical analysis, to verify the calibration of the developed mechanistic model. Figure 1 shows these values, where it can be concluded that the crack activity before overlay can in fact be predicted using the suggested FEM and statistical models.

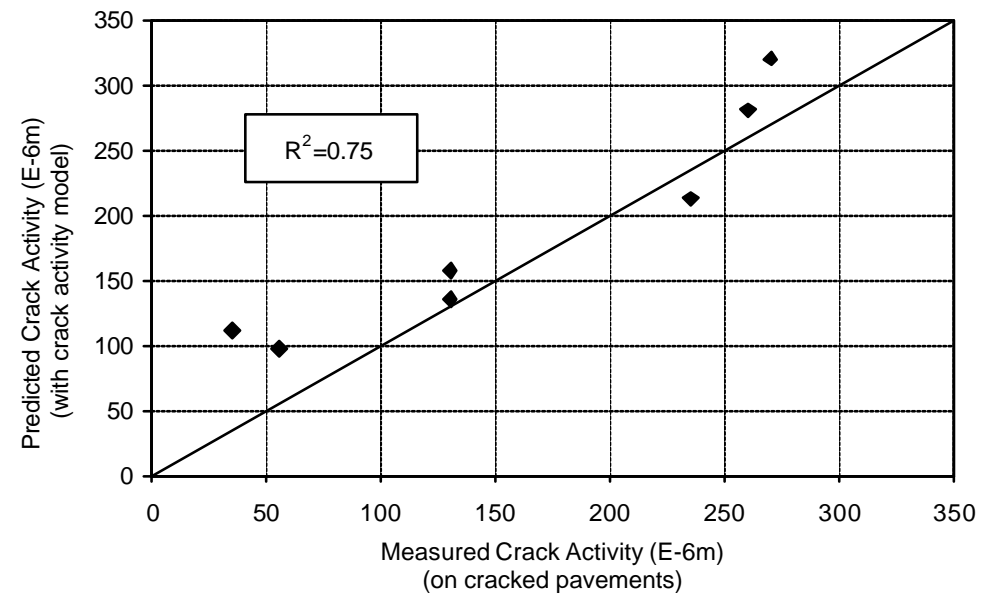

Figure 1. Comparison between Crack Activity Measured In Situ On the 7 Cracked Test Sections in Arizona and California and the Predicted Values Obtained by the Model

\subsection{Measured vs. Predicted Crack Activity after Overlay (ADOT)}

Figure 2 presents the 3D mesh used for the determination of the crack activity after overlay, and the reflective cracking state of stress and strain in the zone above a crack. In this figure, a longitudinal crack is represented for a pavement with 4 layers, i.e., an overlay layer placed on a bituminous cracked layer, a granular layer, and the subgrade layer. 


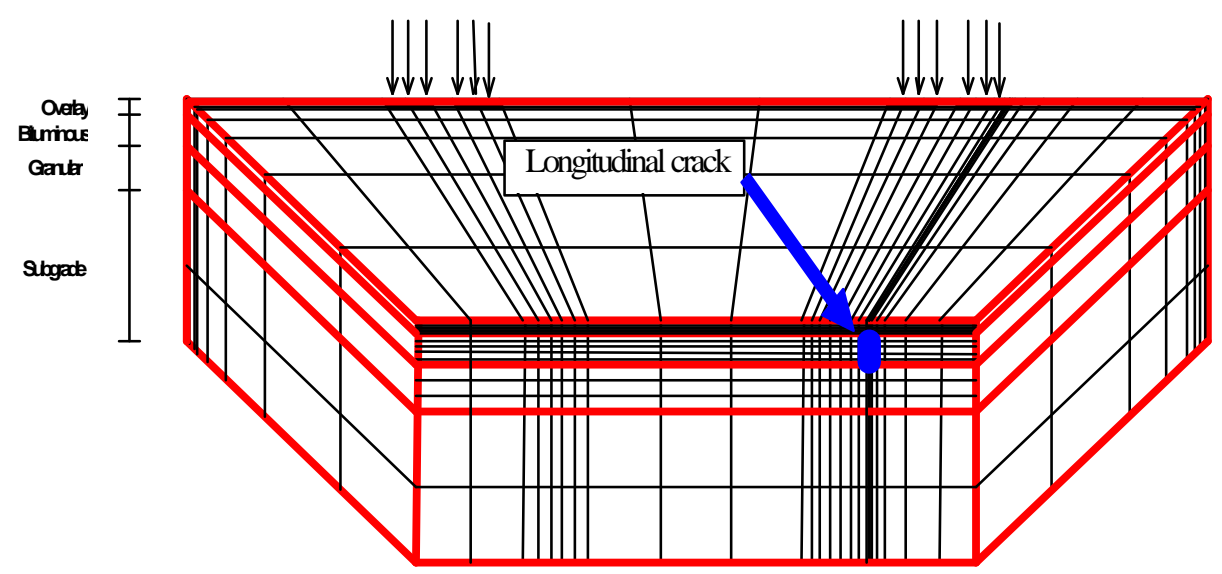

Figure 2.3D Representation of Finite Element Mesh

\subsection{State of Stress and Strain in the Zone above Crack}

To define a statistical model between the state of stress and strain and the pavement properties, a 2D study was carried out where the influence of the shear stress in the zone above the overlay was studied as function of the pavement properties, namely thicknesses and moduli. 4800 pavements were simulated using a 2D mesh on SAP 2000 and the shear stress was obtained by the average shear stress in the elements above the modelled crack. The crack was modelled by a set of elements in the FEM with zero modulus and a width of $1 \mathrm{~cm}$.

Figure 3 represent the typical influence of overlay thickness on shear stress (presented for 8 of the 4800 pavements simulated) where it can be seen that the shear stress follows a power variation with the overlay thickness. The typical influence of the other pavement properties (thicknesses and moduli of the all layers) on shear stress is characterized by a logarithmic variation as expressed in Figure 4 for the overlay modulus (presented for 8 of the 4800 pavements simulated).

Subsequently, a statistical model to define the state of stress and strain in the zone above the crack was developed using the following equations:

$$
\begin{aligned}
& \text { State of Stress }=a *[\text { Overlay thickness }]^{b} \\
& \qquad \begin{aligned}
a & =\prod_{i=1}^{6}\left[a_{1 i} * \ln \left(X_{i}\right)+a_{2 i}\right] \\
b & =\prod_{i=1}^{6}\left[b_{1 i} * \ln \left(X_{i}\right)+b_{2 i}\right]
\end{aligned}
\end{aligned}
$$

Where the $X_{i}$ variables represent the pavement properties and the $a_{i j}$ and $b_{i j}$ the statistical coefficients. 


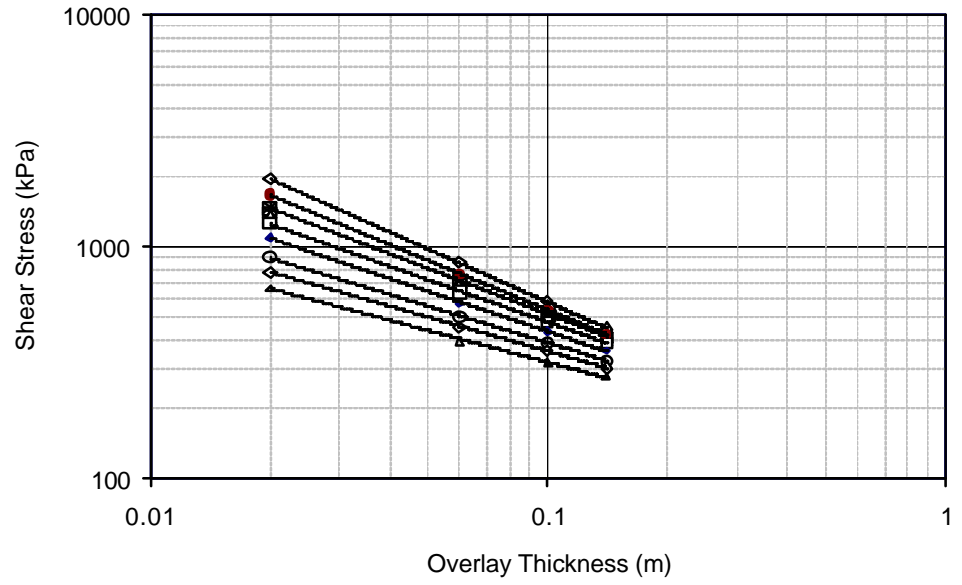

Figure 3. Typical influence of the overlay thickness on shear stress in the zone above crack

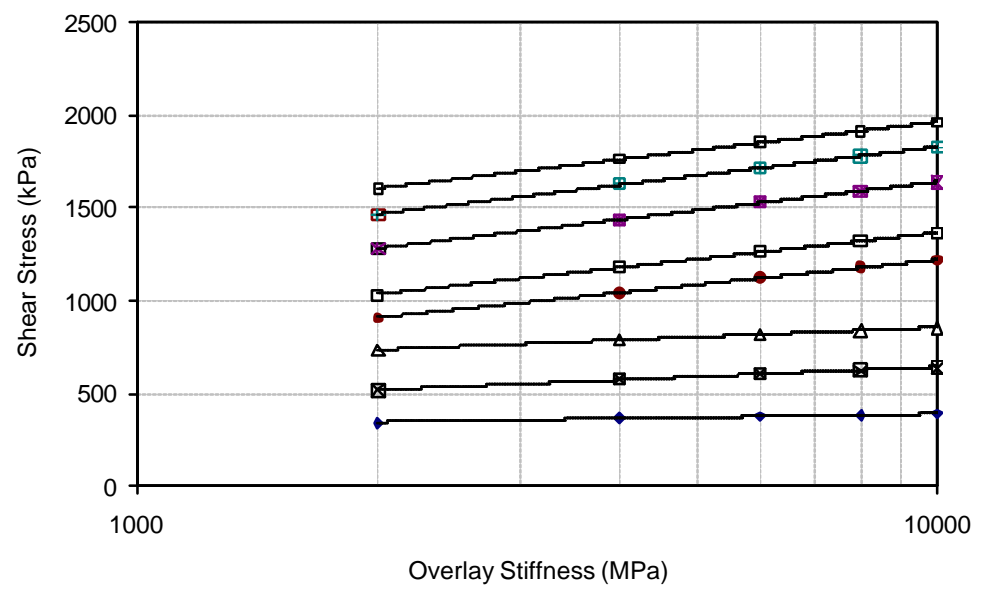

Figure 4. Typical influence of the overlay modulus on shear stress in the zone above crack

The influence of pavement properties on the state of stress and strain was made defining a deviator strain such as the Von Mises stress [REE 2000], which take into account the origin of this phenomenon (stresses and strains in the horizontal and vertical direction). This strain, called the "Von Mises strain" was calculated as expressed in Equation [5]. 


$$
\varepsilon_{V M}=\sqrt{\frac{1}{2}\left(\left(\varepsilon_{1}-\varepsilon_{2}\right)^{2}+\left(\varepsilon_{1}-\varepsilon_{3}\right)^{2}+\left(\varepsilon_{2}-\varepsilon_{3}\right)^{2}\right)}
$$

Where: $\varepsilon_{V M}=$ "Von Mises" strain

$$
\varepsilon_{1}, \varepsilon_{2}, \varepsilon_{3}=\text { Principal strains }
$$

Using the 3D FEM model presented in Figure 2, the $\varepsilon_{V M}$ as the average Von Mises strain in the elements above the crack was calculated for 384 pavements and fitted in a statistical model as the developed in the 2D study.

Thus, the statistical model for the $\varepsilon_{V M}$, is:

$$
\begin{gathered}
\varepsilon_{\mathrm{VM}}\left(1 \times 10^{-6}\right)=a *[\text { Overlay thickness }(m)]^{b} \\
a=\prod_{i=1}^{6}\left[a_{1 i} * \ln \left(X_{i}\right)+a_{2 i}\right] \\
b=\prod_{i=1}^{6}\left[b_{1 i} * \ln \left(X_{i}\right)+b_{2 i}\right]
\end{gathered}
$$

Where: $a_{i j}$ and $b_{i j}$ are coefficients given in Table 1 .

Table 1. Statistical coefficients for the $\varepsilon_{V M}$ model (Equation 4) $\left[R^{2}=0.98\right]$

\begin{tabular}{|c|r|r|r|r|}
\hline$i$ & \multicolumn{1}{|c|}{$a_{1 i}$} & \multicolumn{1}{c|}{$a_{2 i}$} & \multicolumn{1}{c|}{$b_{1 i}$} & $b_{2 i}$ \\
\hline 1 & $-1.038 \mathrm{E}-04$ & $-1.446 \mathrm{E}-01$ & $7.169 \mathrm{E}-03$ & $1.314 \mathrm{E}-01$ \\
\hline 2 & $2.777 \mathrm{E}-01$ & $-4.022 \mathrm{E}+00$ & $9.773 \mathrm{E}-05$ & $-6.368 \mathrm{E}-01$ \\
\hline 3 & $-1.173 \mathrm{E}+00$ & $1.212 \mathrm{E}+01$ & $-4.946 \mathrm{E}-01$ & $7.069 \mathrm{E}+00$ \\
\hline 4 & $1.281 \mathrm{E}+00$ & $5.070 \mathrm{E}-01$ & $3.923 \mathrm{E}-02$ & $2.641 \mathrm{E}+00$ \\
\hline 5 & $-5.160 \mathrm{E}-01$ & $6.964 \mathrm{E}+00$ & $3.265 \mathrm{E}-02$ & $-1.287 \mathrm{E}+00$ \\
\hline 6 & $-1.775 \mathrm{E}-01$ & $2.385 \mathrm{E}+00$ & $1.875 \mathrm{E}-03$ & $-8.167 \mathrm{E}-01$ \\
\hline
\end{tabular}

In the Equations [5] and [6], the variables $X_{i}$, represent the pavement properties namely thicknesses and moduli. These values should be introduced by the order defined in Table 2. This table presents also the minimum and maximum limits for each variable used in the definition of the model.

Table 2. Variables defining the pavement properties in equation 5 and 6

\begin{tabular}{|c|l|c|c|}
\hline$i$ & \multicolumn{1}{|c|}{$X_{I}$} & Minimum & Maximum \\
\hline 1 & Thickness of the existing cracked layer $(\mathrm{m})$ & 0.10 & 0.25 \\
\hline 2 & Thickness of the granular layer $(\mathrm{m})$ & 0.20 & 0.40 \\
\hline 3 & Modulus of the overlay layer $(\mathrm{MPa})$ & 2000 & 10000 \\
\hline 4 & Modulus of the existing cracked layer $(\mathrm{MPa})$ & 2000 & 3500 \\
\hline 5 & Modulus of the granular layer $(\mathrm{MPa})$ & 150 & 450 \\
\hline 6 & Modulus of the subgrade layer $(\mathrm{MPa})$ & 50 & 150 \\
\hline
\end{tabular}


The statistical model developed for the Von Mises strain can predict the values calculated using the 3D FEM as can be observed in Figure 5, where the residual (difference between the strain calculated using the FEM and the predicted by the statistical model) is relatively small for all results.

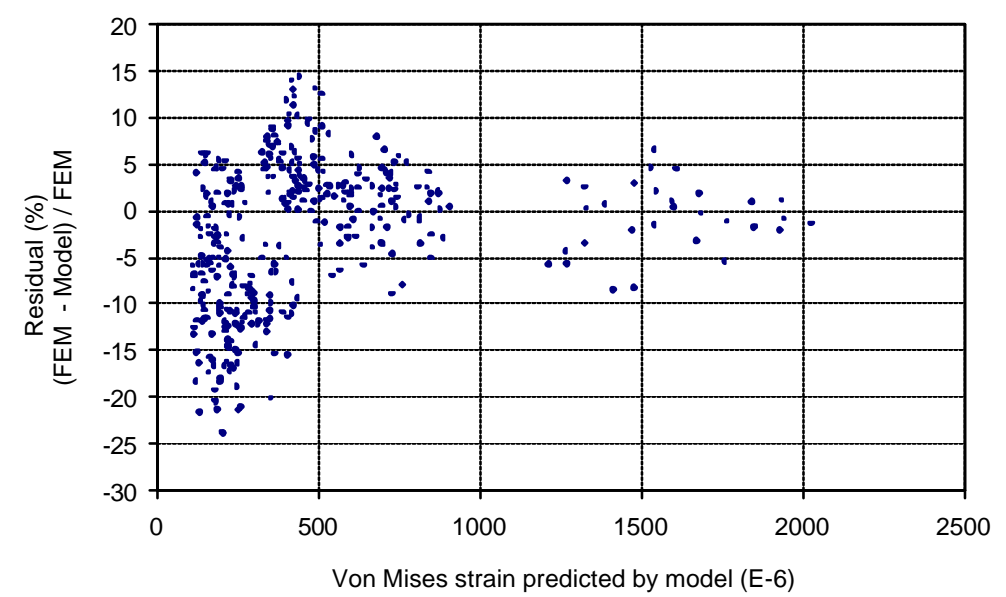

Figure 5. Difference between FEM values and statistical values (residual) for Von Mises strain

\section{Laboratory Tests}

The crushed aggregate used was derived from good quality Portuguese granite. For the conventional HMA mixes, it was graded to meet a dense gradation similar to that used in Arizona and California for neat asphalt mixes. Similarly, the same aggregate was gap-graded in a manner similar to that used in Arizona and California for the (wet process) asphalt rubber mixes [WAY 2000].

The binders used were a PG 70-10 and a PG 64-16 (PEN 35/50) for the neat asphalt mixes. For the AR mixes, the PG 64-16 asphalt cement was interacted with $20 \%$ crumb rubber from California for one binder type, generally referred to as the Arizona "Type A" AR Binder. For the California AR binder, AR-4000 asphalt cement was mixed with California crumb rubber, natural rubber, and extender oil in a manner consistent with routine California work (generally called the "Type B" AR binder).

The binder content for the neat asphalt HMA-DG mix was 5\% and for the AR gap-graded mix (AR-HMA-GG) it was 8\%, consistent with typical Arizona and California mix types and designs. 
Compaction was carried out with a steel roller in a heating mould, in order to maintain compaction temperature (AASHTO PP3-94: Standard Practice for Preparing HMA Specimens by Means of the Rolling Wheel Compactor). The compacted slabs were sawed and cored with the appropriate dimension for each type of test. For determining flexural fatigue life, beam specimens $380 \mathrm{~mm}$ long x 50 $\mathrm{mm}$ thick x $63 \mathrm{~mm}$ wide were used.

Flexural fatigue tests were conducted according to the AASHTO TP 8-94 (Standard Test Method for Determining the Fatigue Life of Compacted HMA Subjected to Repeated Flexural Bending). All tests were carried out at $20^{\circ} \mathrm{C}$ and at $10 \mathrm{~Hz}$ frequency rate of loading.

The flexural fatigue tests conducted in displacement control present the fatigue life expressed as function of the tensile strain. For beam fatigue test conditions subjected to four-point bending, $\varepsilon_{V M}$ can be written as:

$$
\varepsilon_{V M}=\varepsilon_{1}(1+v)
$$

where $v$ is the Poisson ratio.

Thus, the fatigue life as function of $\varepsilon_{V M}$ is presented in Figure 6.

It can be observed that the flexural fatigue life of the AR-HMA-GG mixes under strain control mode is some 10 to 40 times greater than that of HMA-GG mixes.

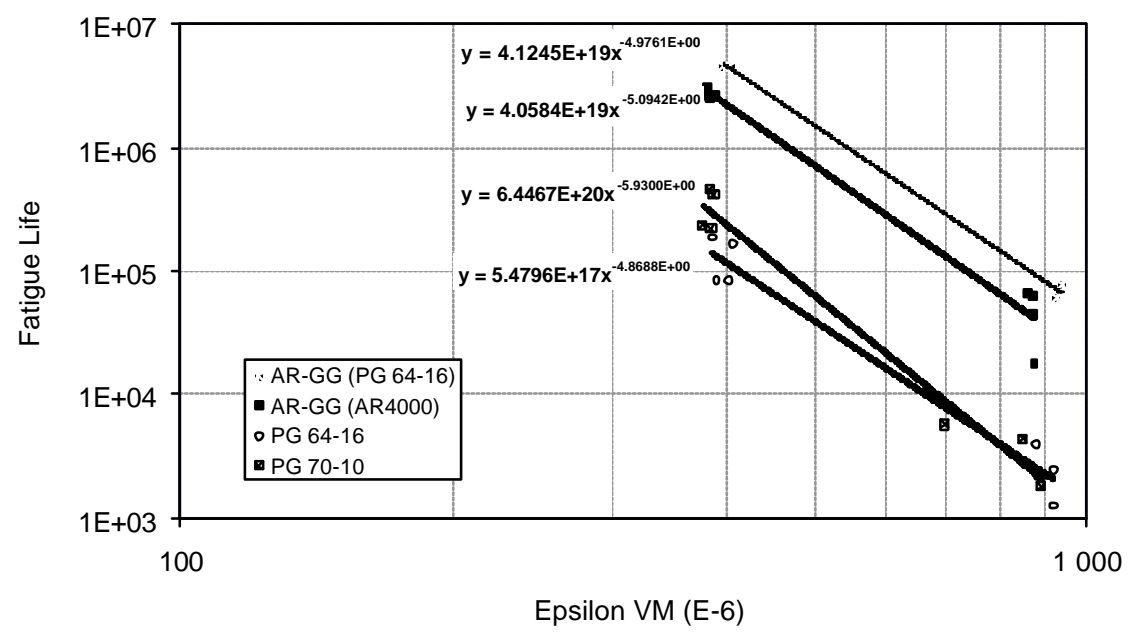

Figure 6. Flexural Fatigue Life as a Function of $\varepsilon_{V M}$ 


\section{Field and Performance Data to Calibrate the Reflective Cracking Model}

An empiricalmechanistic model, such as the one developed in this research effort, requires that predictions of performance are calibrated and adjusted against real field performance. This is a complex and tedious process, and it must be based on reliable and extensive field data. In this project, we were provided with excellent data collected over the years by ADOT.

\subsection{Definition of Cracking Levels}

The percent cracking refers to an ADOT method of expressing the amount and area of cracking developed in the early 1970's [WAY 79]. Percent cracking was developed in English units since it referred to the amount of cracking within a standard area as defined at the AASHTO Road Test in the early 1960's. The standard area is 93.6 square meters (1,000 square feet, AASHTO definition), which represents the lane width of 3.7 meters (12 feet) by 25.3 meters ( 83 feet) of length. By the Arizona method one percent cracking is equal to 6.1 lineal meters (20 lineal feet) of cracking within the standard area. Likewise, 10 percent is 61 lineal meters (200 lineal feet) per the standard area and 100 percent is 610 lineal meters $(2000$ lineal feet) of cracking per standard area. ADOT has used this method of recording the percent of cracking as inventoried at each milepost since about 1972. Values have ranged from as low as no cracks ( 0 percent) to as high as 95 percent. Percent cracking values from ADOT's Pavement Management System annual crack inventory were used as input into the model development.

\subsection{ADOT Data from Selected Test Sections}

ADOT furnished all the data for this research study from thirty test sections throughout the state which included cracking information, layer moduli, layer thicknesses, overlay material properties, aggregate gradation, binder type and binder content. Pavement cracking information (both before and after overlay), average ESALs per year, total predicted ESALs, local temperature information, and recommended PG-grade for the region was also included. The available data is too extensive to be included in this paper.

\subsection{Adjustment Factors for Reflective Cracking Method}

The calibration of the model was made based on an iterative process that is too extensive to be covered in this paper. In general this process involved the development of an aging adjustment factor (AAF), a temperature adjustment factor and a field adjustment factor, which are explained in more detail. 


\subsubsection{The Aging Adjustment Factor (AAF)}

This factor was introduced to capture the effect of aging in the overlay as a function of the maximum air temperature. It has been documented that aging plays a very important role in the performance of an asphalt-bound overlay. Furthermore, over time it will stiffen the overlay (raise its modulus). The magnitude of this effect was also noticed by [RAD 2001], for both the HMA-DG and AR-HMA-GG mixes. In Raad's report it was found that, for the relatively mild climate of the Los Angles area, the AAF was 1.07 for AR-HMA-GG mixes and 1.30 for HMA-DG mixes (over a life span of approximately 10 years).

Figure 7 shows the data from which the AAF was determined, for AR-HMA-GG and HMA-DG mixes respectively.

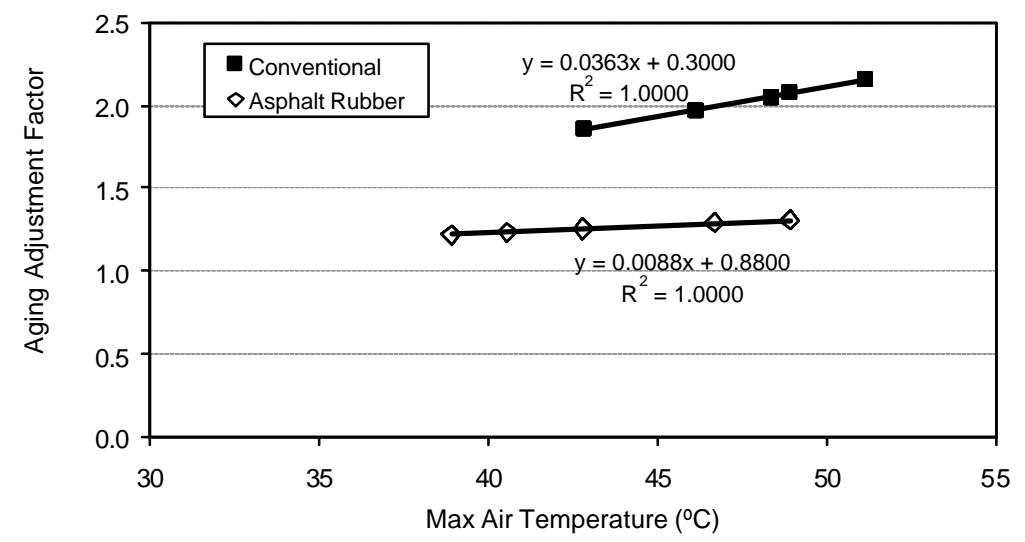

Figure 7. Determination of the Aging Adjustment Factor for Asphalt Rubber and Conventional AC40 binders

Based on the available data, the Aging Adjustment Factor may be expressed as:

$$
A A F=0.0363 * T(\max \text { air })+0.3000
$$

for conventional PG70-10 binders, and

$$
A A F=0.0088 * T(\max \text { air })+0.8800
$$

for asphalt rubber binders.

Further improvements in these relationships are still needed. 


\subsubsection{The Temperature Adjustment Factor (TAF)}

It is well documented that most reflective cracking occurs when lower temperatures occur. The actual phenomenon is very complex. Several mechanisms play a role concurrently:

- Load associated strains occur above a crack each time a vehicle passes over (or near) the crack.

- As the temperature decreases during its daily cycle, it can actually reach a $20^{\circ} \mathrm{C}$ to $25^{\circ} \mathrm{C}$ fall during each day [LUK 2000]. These rapid cooling rates cause overall states of tension in the overlay and are particularly important above the crack. They are also a function of the creep compliance of the material. In fact, the low temperature effects alone can cause greater tensile stresses than those caused by actual passing wheel loads.

- The combination of the two most important effects passing wheel loads on a daily basis above (or near) the crack and the material (overlay) above the crack being under tension due to rapidly decreasing or low temperatures have been identified as the most likely causes of high states of stress and strain above the crack.

- The binders PG grade (and where a particular PG grade is used) does play an important role in the reflective cracking phenomena. For instance, it is believed that if a region calls for a PG grade of XX-10 but in practice a PG grade of XX-32 is used, an overlay placed using the latter of these binders is less likely to crack than if the XX-10 binder is used. Thus to a certain extent, reflective fatigue cracking is associated with thermal fatigue cracking.

It is possible to model all of these effects, but such efforts were beyond the scope of the research project. Nevertheless, every attempt was made to develop a methodology to consider as many effects as possible through the use of adjustment factors, as outlined above. For example, many overlapping low temperature effects are most likely captured globally in the Temperature Adjustment Model (TAF). The adjustments do magnify the magnitude of the $\varepsilon_{V M}$ predicted by the load-associated model to capture these temperature effects. Using data obtained at the ADOT locations selected for this study, it was calibrated for the HMA-DG and AR-HMAGG, as shown in Figure 8. The Reflective Cracking Temperature (RCT) was arbitrarily set as the mean value between the minimum air temperature and the average mean air monthly temperature. 


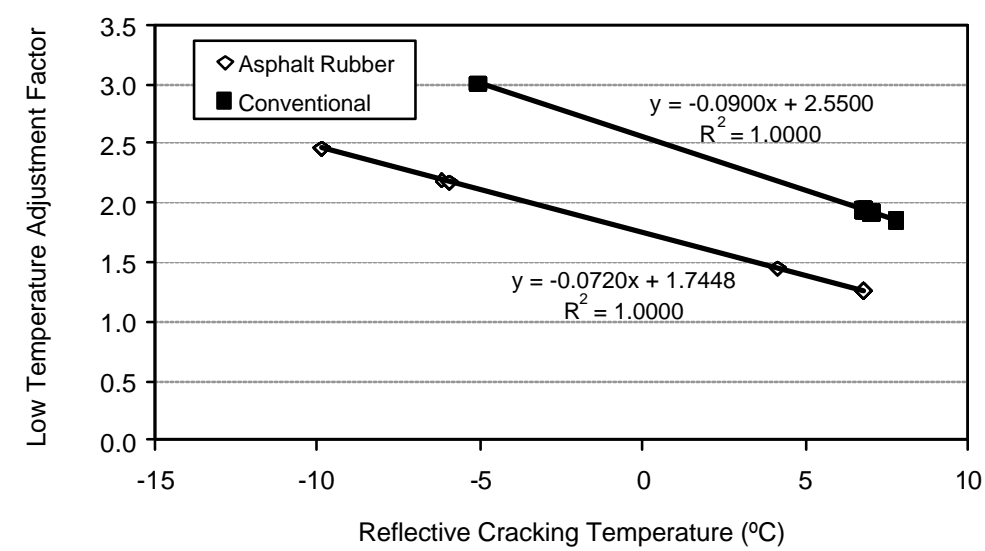

Figure 8. Development of Low Temperature Adjustment Factors for Asphalt Rubber and PG70-10 Neat Asphalt Binders as a Function of Reflective Cracking Design Temperature

It is interesting to notice that the negative effects (i.e., the higher the value of the TAF, the more damaging the effect is) of progressively lower temperatures. Furthermore it is also interesting that the Temperature Adjustment Factor is higher for the AC40 (PG70-10) mixes.

Future improvements in this factor with this approach may depend on the relation between the minimum PG temperature of the binder and the location of the pavement section. However, based on the current data, the following formulae are proposed for conventional AC 40 binders:

$$
T A F=-0.0900 * R C T+2.5500
$$

and for asphalt rubber binders:

$$
T A F=-0.0720 * R C T+1.7448
$$

where:

$$
R C T=T(\min \text { air })+0.5 \times[T(\text { averageair mean monthly })-T(\min \text { air })]
$$

To investigate the validity of the existence of a temperature adjustment factor a 3D FEM analysis was made where 4 pavements (2 with an ARHM overlay and 2 with a DGAC overlay) were subjected to a temperature drop in the bituminous layers. The overlay thickness was set to 4 and $8 \mathrm{~cm}$ and the modulus, at the reference temperature used $\left(23^{\circ} \mathrm{C}\right)$, were $5000 \mathrm{MPa}$ for ARHM and $10000 \mathrm{MPa}$ for DGAC simulating the aging effect in the mixes. The pavement below the overlay is characterized by a cracked layer with $18 \mathrm{~cm}$ and $3000 \mathrm{MPa}$, a granular layer with 15 $\mathrm{cm}$ and $250 \mathrm{MPa}$ and a subgrade with $110 \mathrm{MPa}$ stiffness. The thermal contraction 
coefficients used were $1.4 \times 10^{-5} /{ }^{\circ} \mathrm{C}$ for DGAC and $2.16 \times 10^{-5} /{ }^{\circ} \mathrm{C}$ for ARHM. This study was conducted for 4 temperature drops $\left(-10^{\circ} \mathrm{C},-15^{\circ} \mathrm{C},-25^{\circ} \mathrm{C}\right.$ and $\left.-30^{\circ} \mathrm{C}\right)$. The stiffness of bituminous layers (cracked layer and overlay layer) was changed as function of the temperature decrease and for ARHM is was assumed that the stiffness increases $250 \mathrm{MPa}$ for $1^{\circ} \mathrm{C}$ of temperature decrease. For DGAC it was assumed that the stiffness increases $300 \mathrm{MPa}$ for $1^{\circ} \mathrm{C}$ of temperature decrease as recommended by [PAI 2002].

The results of this analysis in are presented in Figure 9 where the Von Mises stresses are plotted against the temperature drop in both bituminous layers. It can be concluded from analysis of the results that the stress in the zone above the crack, calculated as the average stress in all FEM elements above the crack, increase when the overlay thickness decrease and is greater for DGAC than for ARHM. The stress ratio between the two bituminous mixtures in the overlay is on average 1,22 .

These values indicate the proper trend. If more precise measurements of the change in modulus with temperature are made it is very likely that similar values would be obtained thus validating the TAF concept and range in values. The ratio between the TAF for DGAC and ARHM is, as presented in Figure 8, on average 1,5. This difference can be attributed to other factors that appear in the pavement that are not considered in the FEM analysis. Thus, it can be concluded that the TAF reasonably represents, albeit in a simplified manner, the effect of temperature drop.

\subsubsection{Field Adjustment Factor (FAF)}

The Field Adjustment Factor (FAF) was introduced to relate the predictions obtained using the empirical- mechanistic reflective cracking model with actual (reported and observed) field performance. This value was initially determined following the same steps identified above.

Cracking is only expected when FAF is greater than 1. For FAF values less than 1 , cracking should not have occurred. If the model predicts otherwise, the model is incorrect. It should be noted that the reported percent cracking may go up and down from year to year due to maintenance crack sealing activities which introduces greater scatter in the reported data. 


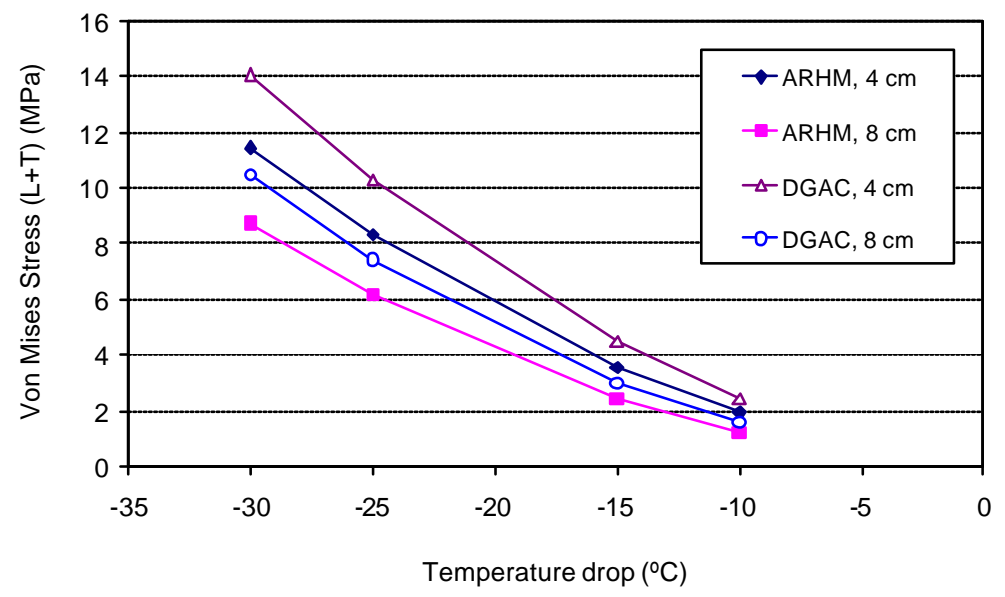

Figure 9. Von Mises stress plotted against pavement temperature drop

Figure 10 shows the relationship between percent cracking and the field adjustment factors obtained for the asphalt rubber sections with gap-graded aggregate gradations (AR-HMA-GG).

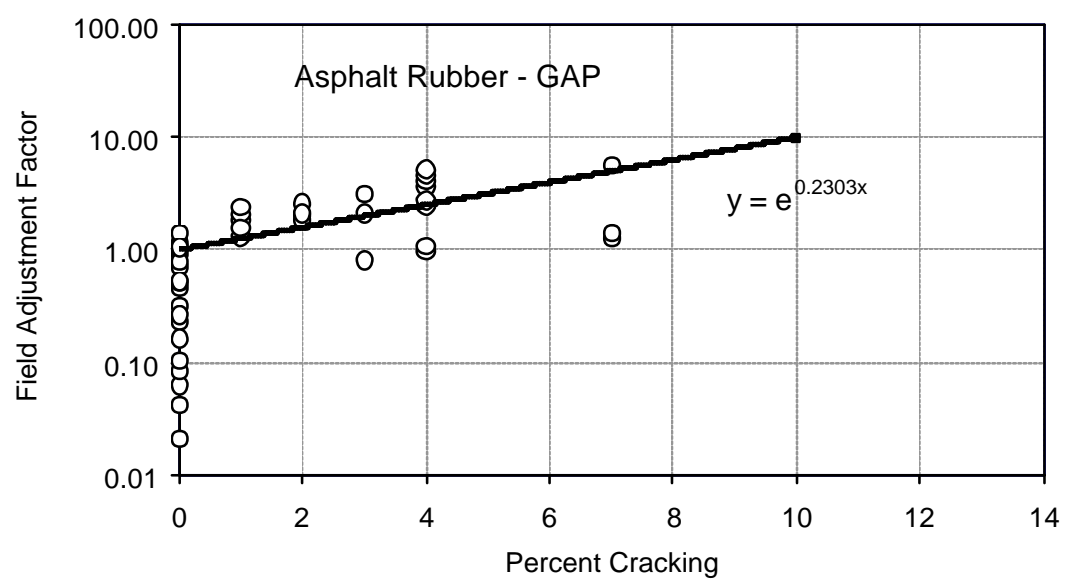

Figure 10. Relationship between FAF and Percent Cracking for Asphalt Rubber Gap-Graded Sections

It can be observed that for the vast majority of points where the FAF was below 1 , no cracks were reported. When cracks were reported by ADOT, the FAF was higher than 1. This follows a clear trend that can be expressed by Equation [15], where $P C$ is the percent cracking. 


$$
F A F=e^{0.2303^{*} P C}
$$

The relationship obtained for the conventional mixes between percent cracking and FAF is presented in Figure 11. It can also be observed in this figure, that when the FAF is less than 1 no cracking is reported. However when cracking occurs, the FAF is always greater than 1 . The same relationship between cracking and FAF encountered for the asphalt rubber mixes also appears to be appropriate for the conventional mixes.

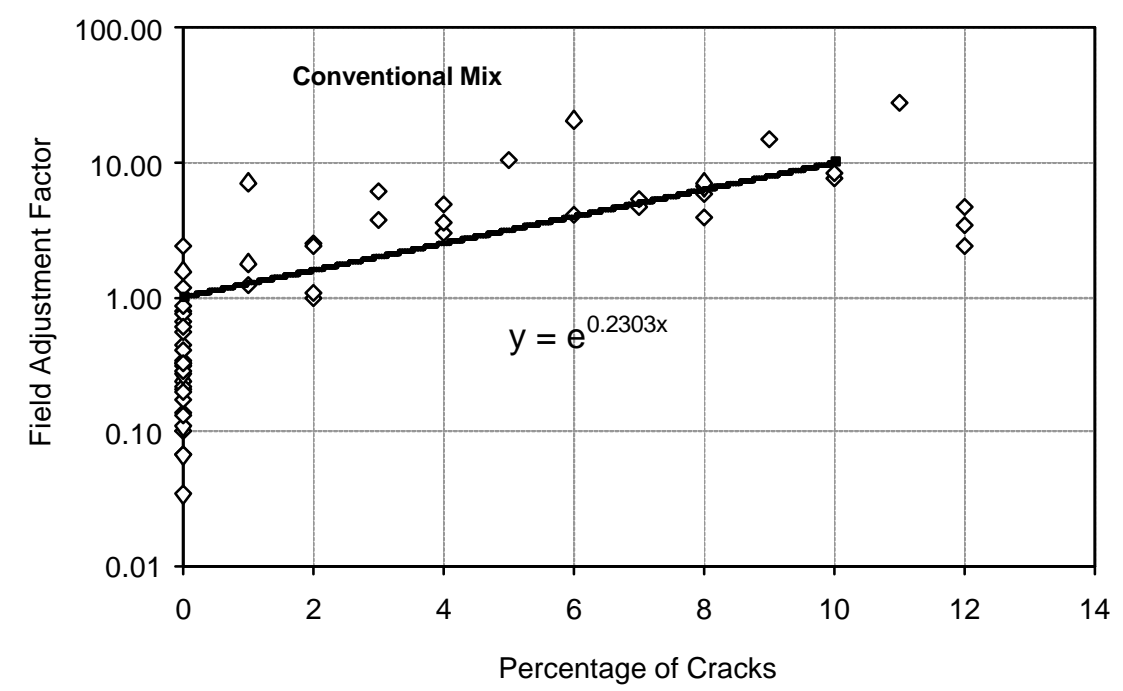

Figure 11. Relationship Between the Field Adjustment Factor and Percentage of Cracks for Conventional Mixes (PG70-10)

The determination of the three factors AAF, TAF and FAF was done simultaneously, iteratively and interactively and the results appeared reasonable. Combining these three factors in a logical fashion involved engineering judgment considerations.

Once all of the values were calculated, another approach was followed to investigate the quality of fit of the proposed reflective cracking model. In this case, the slopes and intercepts for the AAF and TAF were varied in such a manner as to minimize the $\mathrm{R}^{2}$ fit of the model predictions, for all pavements having crack patterns between $1 \%$ and $10 \%$. Figure 12 shows the relationship between the predicted and observed number of ESA Ls to the reported percent cracking. It can be observed that the vast majority of the predictions were very close to the actual observations. If the points represent a pavement section built with an unusual and non-standard cinder 
aggregate (the one with the largest outliers) are removed from the database, $R^{2}$ reaches 0.80 .

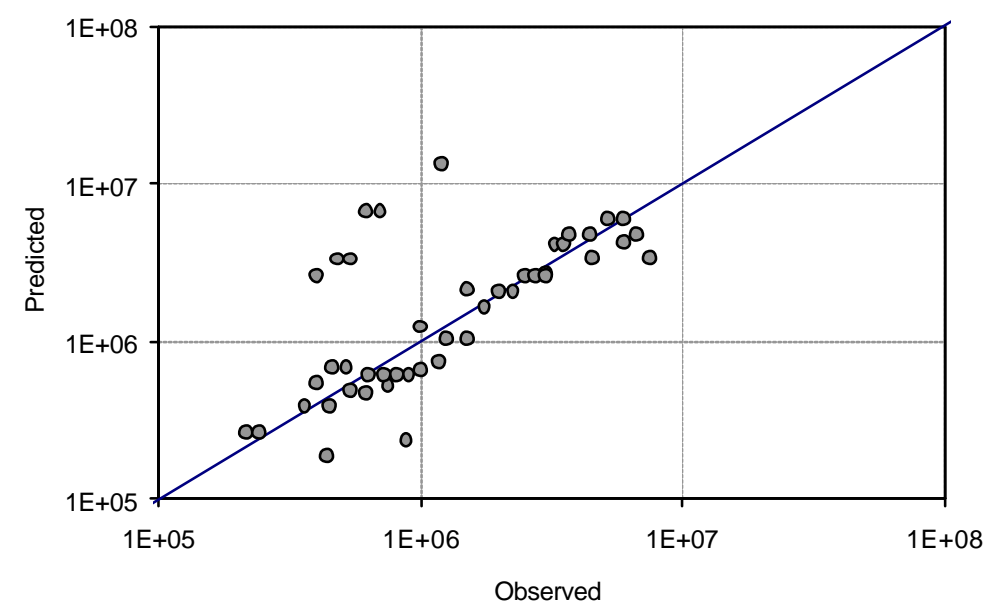

Figure 12. Comparison Between Modeled and Predicted Number of ESALs to the Reported Crack Level for all Pavement Sections in the Database (Conventional and Asphalt Rubber)

From the above considerations and analyses conducted, it can now be seen that the relationships between the Maximum Air Temperatures and the Aging Adjustment Factor, and also between the Reflection Cracking Temperature and the Temperature Adjustment Factor have been adjusted and plotted, as shown in Figures 7 and 8 respectively. This indeed confirms that the trends reported do exist, and that the aging and low temperature effects (captured by the ATF and the TAF respectively) do in fact play an important role in the reflective cracking phenomenon.

In essence, the reflective cracking phenomenon depends on load associated shear loading near the edge of the crack and on two major environmental factors: Aging of the overlay and the tensions over the crack caused when the temperatures drop rapidly during colder winter days.

\section{Proposed Reflective Cracking Design Method}

A methodology is hereby proposed for the design of overlays on existing, cracked flexible pavements to minimize the risk of premature reflective cracking. The method consists of the seven steps presented below. Currently the model has been calibrated for only two materials: Dense graded mixes with PG70-10 binders (HMA-DG) or gap graded mixes with asphalt rubber modified binders (AR-HMAGG). The asphalt rubber binder must be produced using the "wet" process and it must contain approx. 19-20\% crumb rubber. 


\section{Determination of the Moduli and Thicknesses of the Pavement Section Layers}

This can be accomplished using FWD backcalculation methods or other forms of estimating cracked pavement section moduli. Care must be taken in the selection of modulus representative of the most damaged sections. As such, the 90th or 95th percentile of deflections (or backcalculated moduli) should be selected. Coring for determination of layer thicknesses should be carried out as close to the locations where the 90-95th percentile FWD test points were selected.

\section{Determination of Representative Air Temperatures}

The maximum and minimum air temperature determined with the desired reliability should be obtained for the location where the pavement is to be overlaid. Furthermore, it is necessary to compute the mean annual air temperature as proposed by the Shell design method.

The mean annual air temperature is defined as proposed by the Shell design method [CLA 77]. This temperature is a weighted temperature. The weight factor (w-factor) is a function of the mean monthly air temperature (MMAT) and can be obtained by the equation presented in Figure 13 that expresses the Shell chart for this determination.

The weighted mean annual air temperature (w-MAAT) is obtained using the equation from the Figure 14, resulting from the Shell chart.

Thus, the mean annual air temperature, as proposed by the Shell design method (w-MAAT), can be calculated using the Equations [16] and [17].

$$
w-M A A T=7.7068 \operatorname{Ln}(w-\text { factor })+20.257
$$

where:

$$
w-\text { factor }=0.0723 e^{0.1296 M M A T}
$$

where w-factor is the average of w-factors calculated for all 12 months of the year and MMAT is the mean monthly air temperature.

\section{Selection of Design Cracking Percentage}

The percent cracking should be in keeping with that previously discussed. The value selected should be in keeping with an agencies overlay policy. ADOT generally has observed less than five percent cracking over a period of ten years when an asphalt rubber surface mix is used. 


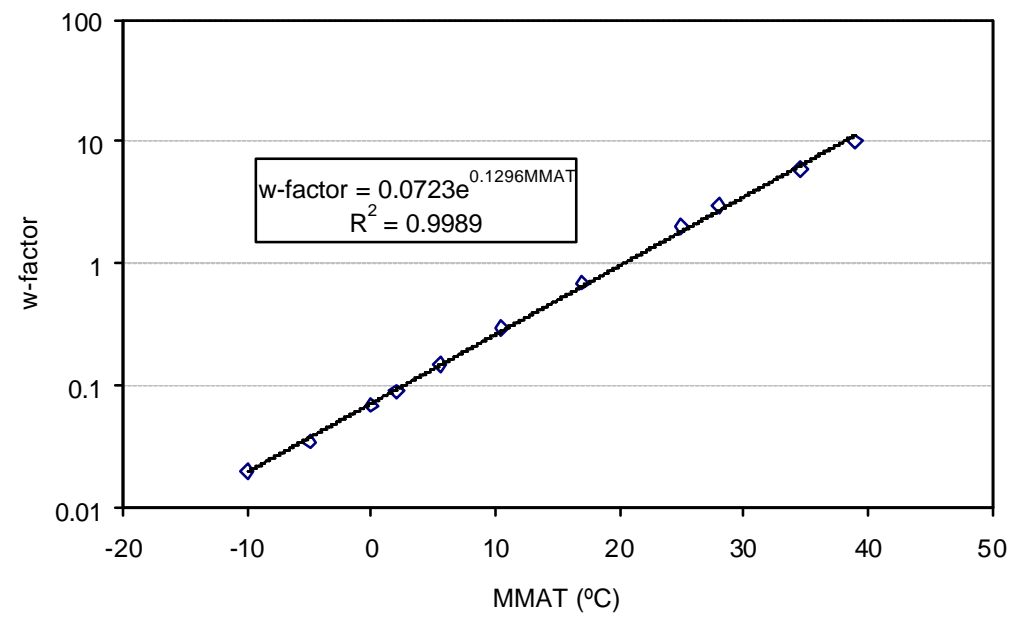

Figure 13. Shell $w$-factor as function of temperature

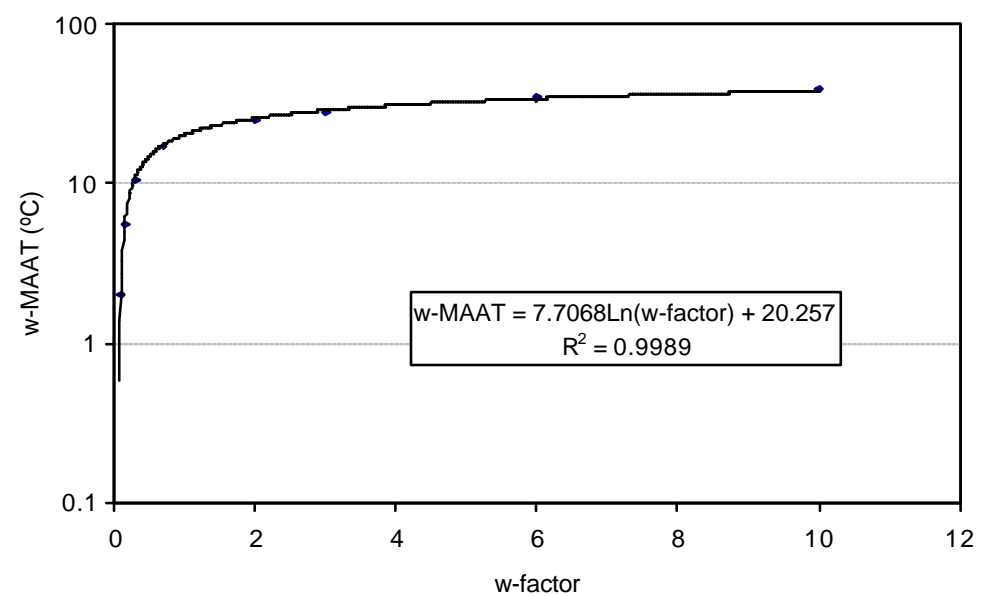

Figure14. Shell $w$-MAAT as function of $w$-factor

\section{Determination of Adjustment Factors}

Several adjustment factors must be calculated for the location where the overlay will be placed and for the desired cracking level at the end of the overlay's design life.

The Aging Adjustment Factor (AAF) is determined from the Equation [10] for conventional PG70-10 mixes, called "HMA-DG" or Equation [11] for asphalt rubber mixed with the gap-graded wet process @ 19\% -20\% binder 
content, called "AR-HMA-GG". Theses are applicable for TMAX temperatures between $35^{\circ} \mathrm{C}$ and $50^{\circ} \mathrm{C}$.

The Temperature Adjustment Factor (TAF) is determined from Equation [12] for HMA-DG mixes or Equation [13] AR-HMA-GG mixes (wet process only). This method is applicable for Reflective Cracking Temperatures (RCTs) between $-10^{\circ} \mathrm{C}$ and $+10^{\circ} \mathrm{C}$.

The Field Adjustment Factor (FAF) is computed from the Equation [15]

\section{Selection of Overlay Material Modulus}

Two types of materials for the overlay may be selected: Conventional HMADG or AR-HMA-GG, with the rubberized binder prepared through the wet process @ 19-20\% crumb rubber in the binder.

For these materials, the modulus and flexural fatigue life are obtained through flexural fatigue tests, as presented in Figure 6. Other moduli can be computed and introduced in the method based on actual tests performed on other types of materials. However, it must be assumed that the Temperature Adjustment Factor or the Aging Adjustment Factor will either be identical to that of the HMA-DG material or the AR-HMA-GG material.

\section{Determination of the Design Value, $\varepsilon_{V M}$}

The modulus of the overlay must be multiplied by the computed Aging Adjustment Factor. With the modulus and thickness for each layer, the $\varepsilon_{V M}$ value for the overlay is determined using Equations [6], [7] and [8].

The $\varepsilon_{V M}$ value obtained through these equations must be multiplied by $86 / 132$ (to convert $\varepsilon_{V M}$ from $130-\mathrm{kN}$ to $80-\mathrm{kN}$ axle loads), and also by the Temperature Adjustment Factor (TAF). The value obtained in this process is thus the design $\varepsilon_{V M}$.

\section{Determination of Design ESALs}

Using the appropriate flexural fatigue equation as introduced in Figure 6, determine the number of ESALS that can be sustained by the overlay prior to the onset of reflective cracking, i.e.:

$$
E S A L s=4.1245 E 19 *\left[\varepsilon_{V M}\left(1 \times 10^{-6}\right)\right]^{-4.9761}
$$

for asphalt rubber binders derived through the wet process, with a $19 \%-20 \%$ binder content and a gap-graded mix, or:

$$
E S A L s=6.4467 E 19 *\left[\varepsilon_{V M}\left(1 \times 10^{-6}\right)\right]^{-5.93}
$$


for conventional PG70-10 binders and a dense graded mixes.

Multiply the design ESAL by the FAF computed in Step 4. The resulting number should represent the number of ESALs required for the overlay to reach the selected percentage of cracking.

Other fatigue curves can be determined and used by this method, based on actual flexural fatigue tests performed on the specific asphalt (whether conventional or modified) material type proposed with due consideration to all adjustment factors.

A methodology is hereby proposed for the design of overlays on existing, cracked flexible pavements to minimize the risk of premature reflective cracking. The method consists of the seven steps presented below. Currently the model has been calibrated for only two materials: Dense graded mixes with PG70-10 binders (HMA-DG) or gap graded mixes with asphalt rubber modified binders (AR-HMAGG). The asphalt rubber binder must be produced using the "wet" process and it must contain approx. 19-20\% crumb rubber.

\section{Example of Application}

Several examples of the applicability of this method are introduced and shown in Figure 16. An EXCEL spread sheet was created with all the formulas presented in this paper. The input values are entered in column $\mathrm{C}$ and the graph regenerates itself for the new pavement and environment conditions selected. From the demanded traffic the required thickness can be determined for conventional and asphalt rubber mixes. Generally in most cases the asphalt rubber mix will give an overlay about half as thick as the conventional mix for the same design traffic level.

\section{Conclusions}

This research project successfully completed lab and fieldwork to develop a mechanistic empirical method to design hot mix asphalt overlays to resist reflective cracking. The specific design method is good for dense graded asphalt hot mixes and gap graded asphalt rubber hot mixes used in Arizona. It probably can also be applied to Southern California and Western Texas.

The generalized approach is applicable to any type of asphalt hot mix provided the fatigue properties of the mix are determined, the in place FWD deflections and the history of cracking is available. 


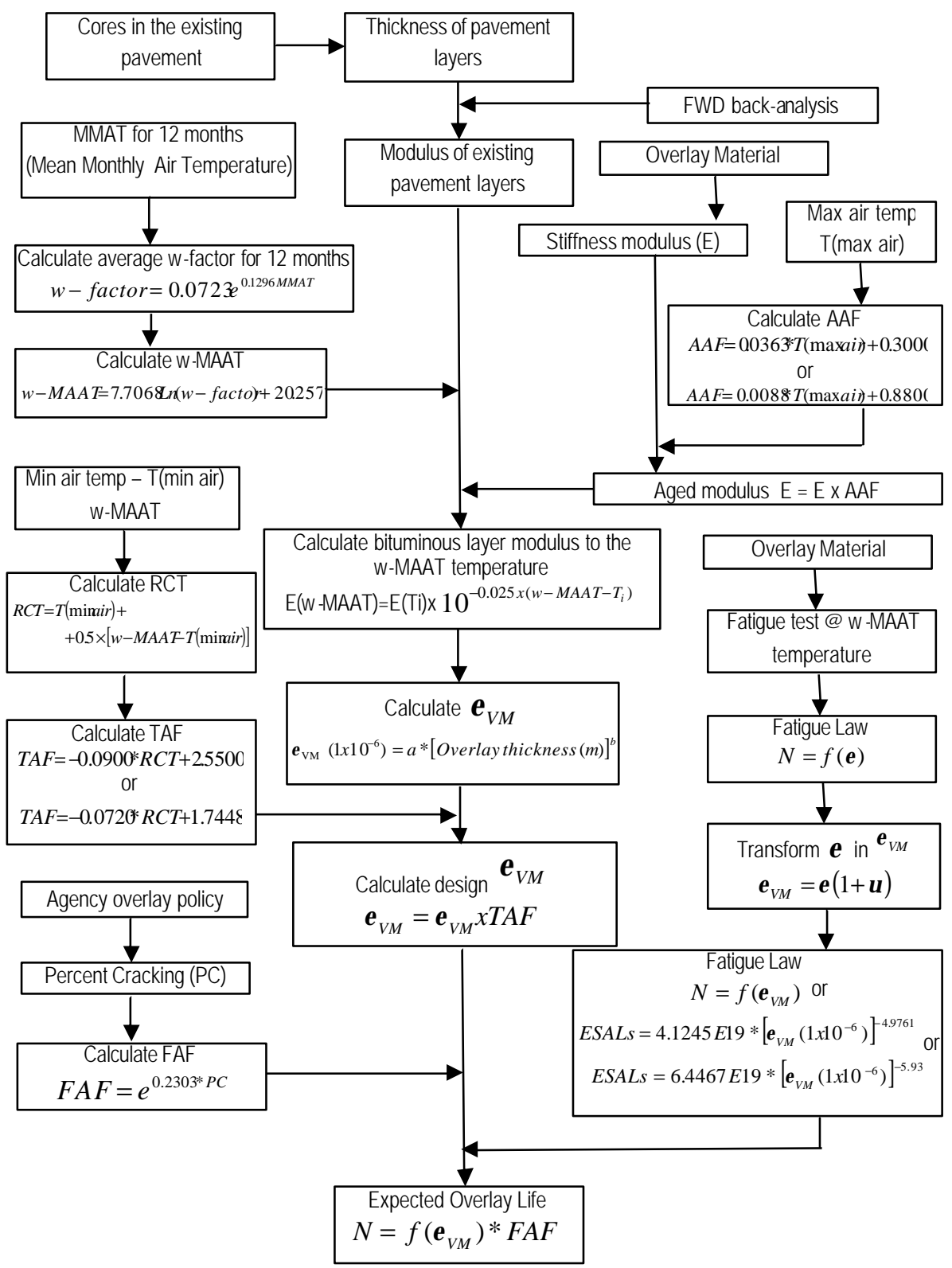

Figure15. Flowchart of the proposed overlay design method 


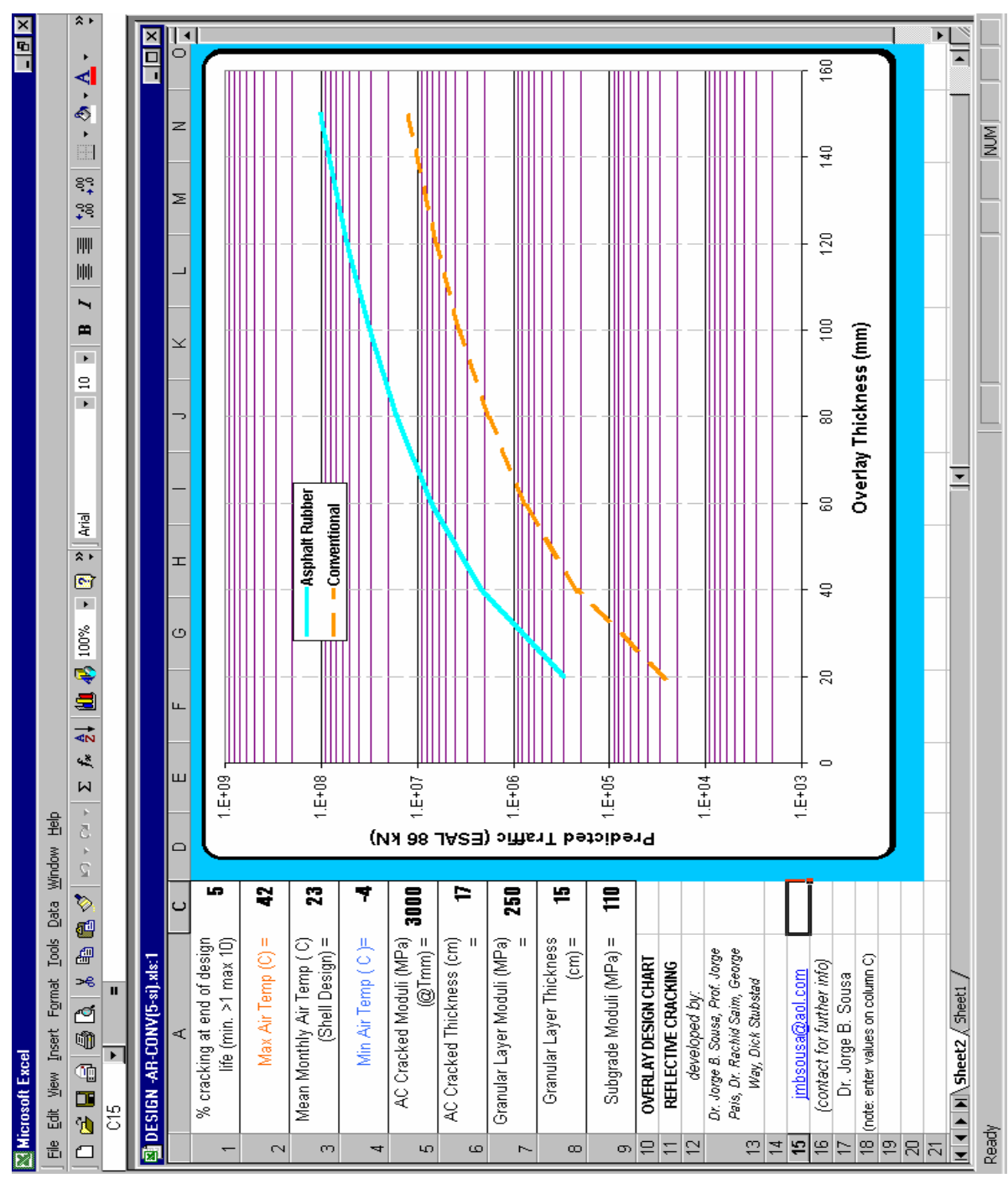

Figure 16. Applicability of reflective cracking model implement in an EXCEL sheet

The following are the main technical findings of the study:

- The Falling Weight Deflectometer can be used in place of the Crack Activity Meter to represent the degree of vertical crack movement [SOU 2001]. 
- The 3D linear elastic model based on the SAP2000 software was used to model the development of average shear stresses above the crack zone as well as to model the crack activity before and after the overlay.

- Field validation showed excellent correlation between the values of the crack activity measured and predicted thus offering a high level of reliability to the value of the average shear stress predicted by the model.

- Statistical models were developed in conjunction with an Excel spreadsheet based on the results of more than 15000 finite element computations. The statistical model and spreadsheet eliminates the need for running a tedious and complex 3D FEM computer program.

- The Finite Element Method (FEM) was successfully used to develop a statistical mathematical model to derive the amount of thickness needed to control reflective cracking.

- The statistical model indicated that the crack width did not appear to relate to the amount of overlay to control a reflective crack.

- The statistical model confirms that asphalt rubber mixes have a much higher capability to resist reflective fatigue cracking. Also, the reduction in overlay thickness by a factor as large as one half is substantially validated by this study [SOU 2001].

There is still research that needs to be completed which includes further improvement of the adjustment factors and the effect of cold temperature shrinkage.

\section{ACKNOWLEDGEMENTS}

The authors greatly acknowledge the cooperation provided by the Rubber Pavements Association, by the Arizona Department of Transportation, by the California Department of Transportation, and by Junta Autónoma de Estradas (the Portuguese Road Directorate). The authors also appreciate the contributions made by Dr. Shakir Shatnawi of Caltrans in the development of this methodology and Ali Zareh of ADOT.

\section{References}

[SOU 2001] Sousa, J.B., Pais, J.C., Saim, R., Way, G.B., Stubstad, R,N, "Development of a Mechanistic Overlay Design Method Based on Reflective Cracking Concepts", Rubber Pavements Association/ADOT, August, 2001.

[WAY 79] Way, G.B., "Prevention of Reflection Cracking Minnetonka-East", ADOT, Report Number 1979GWI, August 1979.

[SCO 89] Scofield, L.A., "History, Development, and Performance of Asphalt Rubber at ADOT”, Report Number AZ-SP-8902, December 1989. 
[WAY 89] Way, G.B., "Flagstaff I40 Overlay Project", 4R Conference \& Road Show, Cincinnati, Ohio, December 1989.

[WAY 2000] Way, G.B., "Flagstaff 140 Asphalt Rubber Overlay Project Nine Years of Success", Transportation Research Board, $78^{\text {th }}$ Annual Meeting, Washington D.C., January 2000.

[REE 2000] Rees, David W.A. "Mechanics of Solids and Structures" Imperial College Press; 1st edition. 2000.

[LUK 2000] Lukanen, E.O., Stubstad, R.N. and Briggs, R.C. "Temperature Predictions and Adjustment Factors for Asphalt Pavement", Federal Highway Administration, Publication No. FHWA-RD-98-085, Washington, DC, June 2000.

[RAD 2001] Radd, L., Saboundjian, S. and Minassian, G. “ Field Aging Effects on the Fatigue of Asphalt Concrete and Asphalt-Rubber Concrete", Transportation research Board, Annual meeting CR Rom, Paper No. 01-3097, Washington, DC, January 2001.

[PAI 2002] PAIS, Jorge C., PEREIRA, Paulo A.A., "Temperature influence on bituminous mixtures stiffness (in portuguese)", $2^{\circ}$ Portuguese Road Congress, Lisbon, 2002.

[CLA 77] Claussen, A.I.M., J.M. Edwards, P. Sommer \& P. Ugé. "Asphalt Pavement Design. The Shell Method", Proceedings, Fourth International Conference on the Structural Design of Asphalt Pavements, Vol. I, Ann Arbor, 39-74, 1977. 